\title{
Identification of Castasterone from a Yeast (Saccharomyces cerevisiae) Strain, WAT21
}

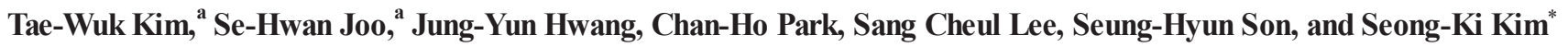 \\ Department of Life Science, Chung-Ang University, Seoul 156-756, Korea.*E-mail: skkimbio@cau.ac.kr \\ Received December 21, 2009, Accepted April 8, 2010
}

Key Words: Brassinosteroids, Saccharomyces cerevisiae, WAT21, Castasterone

Plant hormones are endogenous chemical signals that control a variety of phenomena intrinsic to the growth and development of plants. Currently, several natural and synthetic plant hormones of physiological relevance are used commercially in agriculture as growth regulators, pesticides, and herbicides. ${ }^{1}$ The steroidal plant hormones, known as the brassinosteroids (BRs), regulate a variety of growth and development functions in plants at very low concentrations $\left(>10^{-9} \mathrm{M}\right){ }^{2}$ and thus the BRs are considered promising candidates for a broad range of commercial agricultural applications. However, the high cost required to prepare BRs for such applications remains a problem that must be solved before the commercial use of BRs in agriculture can be realized. In an effort to overcome this problem, we have investigated the possible production of BRs in microbial organisms, which ultimately resulted in the identification of a biologically active BR, castasterone (CS, Fig. 1), in the WAT21 yeast strain. Herein, we report the characterization and metabolism of CS in the yeast cell.

Yeast cells (WAT21) were cultured in a minimal nutrition medium (yeast nitrogen base $6.7 \mathrm{~g} / \mathrm{L}$, D-glucose $20 \mathrm{~g} / \mathrm{L}$ ). After extraction with $80 \% \mathrm{MeOH}$, the BRs in the cultured cells were

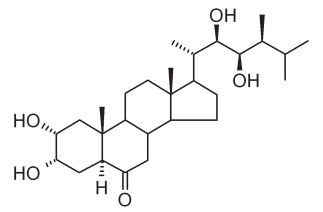

CS

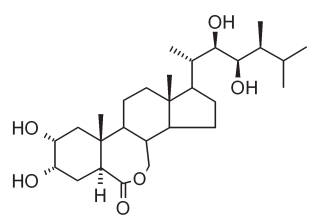

BL

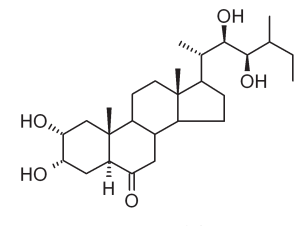

26-NorCS

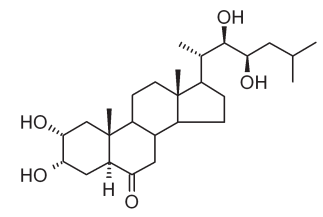

28-NorCS
Figure 1. Structure of BRs mentioned in this study. purified by solvent partitioning, column chromatographies, and a reversed-phase HPLC. In the rice lamina inclination bioassay, ${ }^{3}$ fractions 22 and 23 exhibited biological activity. The fractions were combined, and derivatized with methaneboronic acid $(2 \mathrm{mg})$ in pyridine $(2 \mathrm{~mL})$, then analyzed via capillary GC-MS.

As summarized in Table 1, the bismethaneboronate (BMB) of the active compound in HPLC fractions 22 and 23 yielded a molecular ion at $\mathrm{m} / \mathrm{z} 512$ and prominent ions at $\mathrm{m} / \mathrm{z} 357 / 358$, 327,287 , and 155; these are identical to those derived from authentic CS BMB. The GC retention time of BMB of the compound was also identical to that of CS BMB, thus allowing us to identify the compound as CS, and verifying the occurrence of a BR-namely, CS-in the yeast cells (Table 1). The endogenous amount of CS in the yeast cell calculated using $\left[26,28{ }^{2} \mathrm{H}_{6}\right]-\mathrm{CS}$ as an internal standard was $0.2 \mathrm{ng} / \mathrm{g}$ fresh weight (Fig. 2A).

Although the presence of a CS was demonstrated in the yeast cells, the possible contamination of BRs from the culture media remains. In order to exclude this possibility, the presence of BRs in the culture medium was evaluated. To this end, $2 \mathrm{~L}$ of the medium was extracted and purified in accordance with the same methods as described above. Following HPLC, the fractions corresponding to those of CS were analyzed via GC-MS and GC-Selected ion monitoring (SIM). Even in GC-SIM analysis, no trace amounts of CS were detected in the medium, thereby demonstrating that the medium harbored no BRs. It is, therefore, evident that the CS identified from the yeast cells had been generated by the yeast cell itself.

In plants, $\mathrm{CS}$ is known to be not only a bioactive BR, but also a biosynthetic precursor for the more biologically active $\mathrm{BR}$, brassinolide (BL). In order to determine whether the yeast cells were capable of converting CS to BL, a cell-free convertsion of CS to BL was conducted. Prior to the enzyme assays, the presence of CS was assessed in the crude enzyme solutions. GC-MS/selected ion monitoring (SIM) analyses demonstrated no CS in the solutions (data not shown), thereby indicating that the solutions harbored no detectable quantity of CS. Therefore, non-labeled CS and NADPH (4.8 $\mathrm{mM}$ ) were added to the en-

Table 1. Identification of CS in a yeast strain WAT21 by GC-MS

\begin{tabular}{ccc}
\hline Compound & $\mathrm{Rt}^{a}$ on GC & Prominent ion $^{b}(\mathrm{~m} / z$, relative intensity) \\
\hline CS in yeast & 32.10 & $512(\mathrm{M}+, 80), 441(20), 399(18), 358(33), 327(12), 287(32), 155(100)$ \\
Authentic CS & 32.10 & $512(\mathrm{M}+, 78), 441(19), 399(17), 358(30), 327(11), 287(34), 155(100)$ \\
\hline
\end{tabular}

${ }^{a}$ Rt: Retention time (min). ${ }^{b}$ The samples are analyzed by a capillary GC-MS as BMB.

${ }^{\mathrm{a}}$ Authors are equally contributed. 
Table 2. GC-MS data for 28-norCS, 26-norCS and a CS metabolite in a yeast strain WAT21.

\begin{tabular}{ccl}
\hline Compound & $\mathrm{Rt}^{a}$ on GC & \multicolumn{1}{c}{ Prominent ion $^{b}(\mathrm{~m} / \mathrm{z}$, relative intensity $)$} \\
\hline CS metabolite & 31.60 & $498(\mathrm{M}+, 100), 483(8), 399(3), 358(10), 328(10), 287(33), 141(54)$ \\
Authentic 28-norCS & 31.41 & $498(\mathrm{M}+, 100), 483(8), 399(10), 358(15), 328(9), 287(38), 141(51)$ \\
Authentic 26-norCS & 31.60 & $498(\mathrm{M}+, 100), 483(8), 399(10), 358(27), 328(11), 287(42), 141(93)$ \\
\hline
\end{tabular}

${ }^{a}$ Rt: Retention time (min). ${ }^{b}$ The samples are analyzed by a capillary GC-MS as BMB.
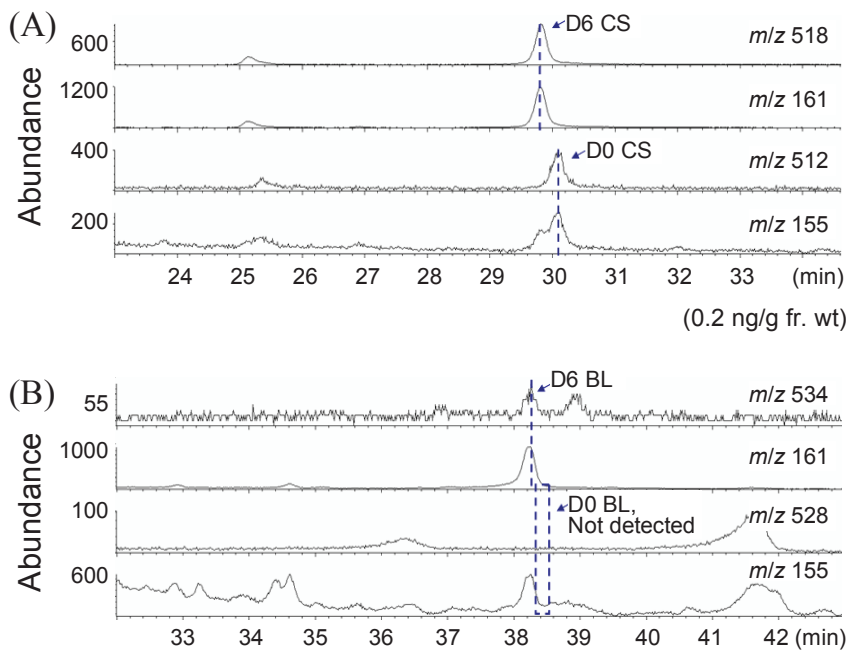

Figure 2. Quantitative analysis of CS and BL in a yeast strain WAT21 by GC-MS.

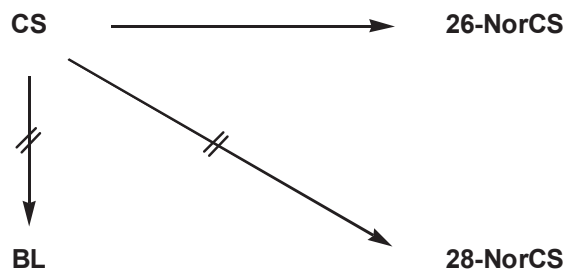

Figure 3. Metabolism of CS in a yeast strain WAT21.

zyme solutions as a substrate and a cofactor, respectively, in order to characterize the metabolism of CS in the enzyme solution. After $30 \mathrm{~min}$ of incubation at $37{ }^{\circ} \mathrm{C}$, the assay mixtures were extracted with ethyl acetate. Further purification was carried out by Sep-Pak C18 cartridge and the same reversed-phase HPLC mentioned above. HPLC fractions 14 and 15, which evidenced biological activity in the rice lamina inclination assay, were combined and then analyzed by GC-MS/-SIM after methaneboronation.

In GC-MS, the bismethaneboronate (BMB) of a CS metabolite exhibited a molecular ion at $\mathrm{m} / \mathrm{z} 498$ and the most abundant ion at $m / z 141$ (Table 2) - this was attributed to the fission of C20/C22 which was reduced in mass by 14 units as compared with CS BMB, implying that a methyl in CS had been eliminated in the metabolite. Therefore, the metabolite was tentatively identified as either 26-norCS or 28-norCS. In GC-MS, the BMB of 26-norCS and 28-norCS generated basically the same mass spectra, but their retention time on GC clearly differed (Table 2). The GC retention time of the BMB of the metabolite was equal to that of 26-norCS BMB, thereby indicating that 26-norCS is a metabolite of CS in the yeast cells (Fig. 3). In the same HPLC, synthetic BL was also eluted in fractions 14 and 15. Therefore, the presence of BL in the fractions was evaluated via GC-MS/SIM analysis. However, not even trace amounts of BL were detected by GC-MS, nor by GC-SIM; this implies that the yeast WAT21 cells harbor no enzymes capable of the conversion of CS to BL (Fig. 2B).

BRs have now been identified in at least 27 families of seed plants (angiosperm and gymnosperm), one pteridophyte, one bryophyte, and one green alga, showing that BRs are fairly ubiquitous plant hormones over the entirety of the plant kingdom. ${ }^{4}$ The results of this study showed that the yeast strain, WAT21, generates steroidal plant hormones, or BRs. This is the first example of the detected presence of BRs in non-plant cells. In accordance with the view that yeast is generally a more primitive living organism than lower plants, BRs are generally thought to be well-conserved ancestral steroidal compounds that predate the evolution of plants. The results of this study demonstrate that WAT2 1 cells generate CS but not BL, thereby implying that $\mathrm{CS}$ is the end product of BR biosynthesis in the yeast cells. Similarly, lower plants produce CS, but not BL. In higher plants, BL is synthesized in reproductively growing tissues such as the pollen, seed, fruit, and flower. ${ }^{4}$ This suggests that the biosynthetic reaction that converts $\mathrm{CS}$ to $\mathrm{BL}$ is an acquired reaction in the evolutionary process from non-seed (flower) plants to seed (flower) plants in the plant kingdom. In this study, we determined that CS is a catabolite of 26-norCS. This deactivation reaction has been noted not only in lower plants but also in higher plants, ${ }^{5}$ thus implying that deactivation by C26-demethylation of active BRs is an ancestral reaction for the maintenance of endogenously active BRs in plant evolution.

WAT 21 is a transgenic yeast strain expressing a gene of Arabidopsis NADPH dependent cytochrome $\mathrm{P} 450$ reductase 2 (ATR2). ${ }^{6}$ In the presence of ATR2, Arabidopsis cytochrome $\mathrm{P} 450 \mathrm{~s}$ can be expressed in yeast cells. via this heterologous expression technique, a number of plant cytochrome P450s have already been expressed in WAT2 1 cells, and their enzyme activities have been characterized previously. ${ }^{7}$ In BRs biosynthesis, two cytochrome P450s, designated 85A1 and 85A2, have been successfully expressed in WAT21 cells, resulting in characterization of $85 \mathrm{~A} 1$ and $85 \mathrm{~A} 2$ as BR oxidase and BR oxidase/BL synthase, respectively, to mediate the final two steps in the generation of active BRs, CS, and BL. ${ }^{8}$

The amount of endogenous CS in the WAT2 1 cells was 0.2 $\mathrm{ng} / \mathrm{g}$ fresh weight-this level is comparable to the levels detected in plants. ${ }^{9}$ This suggests that the development of yeast strain(s) for mass production of BRs might be advantageous, and should be pursued. In light of the fact that BR biosynthesis involves several oxygen incorporation reactions that are mediated by 
cytochrome P450s, the co-expression of the cytochrome P450s involved in BRs biosynthesis in WAT21 cells might constitute a promising method by which the yield of BRs in the WAT21 yeast cells could be augmented.

The physiological function and biosynthesis of BRs in WAT21 yeast cells remains to be clearly elucidated. Additionally, it remains unclear as to whether or not the wild-type yeast, Saccharomyces cerevisiae, can generate BRs. Anyway, the identification of CS in WAT21 yeast cells provides us with an important hint for the possible production of BRs in microbial organisms, because gibberellin, primarily in the form of gibberellic acid, is already mass-produced in the fungus, Gibberella fujikroi, for use in commercial agricultural applications. ${ }^{10}$

\section{Experimental Section}

Purification of BRs in yeast cells. Yeast cells $(100 \mathrm{~g})$ were extracted with $80 \%$ methanol $(1 \mathrm{~L} \times 3)$ followed by chloroform $(1 \mathrm{~L} \times 3)$. After the addition of deuterium-labeled CS $\left(\left[26,28-{ }^{2} \mathrm{H}_{6}\right]-\mathrm{CS}\right)$ as an internal standard for quantification analysis, the chloroform soluble extract was partitioned between $n$-hexane and $80 \%$ methanol. The $80 \%$ methanol soluble fraction was subsequently partitioned between phosphate buffer $(0.1 \mathrm{M} \mathrm{pH} 7.8,1 \mathrm{~L})$ and ethyl acetate $(1 \mathrm{~L} \times 3)$. The obtained neutral ethyl acetate soluble fraction was subsequently purified via silica (20 g) column chromatography eluted with a chloroform-methanol mixture $(0,1,2,3,4,5,6,7,8,9,10$ and $50 \%$ methanol $200 \mathrm{~mL}$ each). The 4, 5, and $6 \%$ methanol in chloroform fractions were combined, then loaded on a SepPak C18 cartridge eluted with aqueous methanol (40, 50, 60, 70, 80, 90 and $100 \%$ methanol $5 \mathrm{~mL}$ each). The 70 and $80 \%$ methanol fractions were purified via reversed-phase HPLC (Senshu Pak $\mathrm{C} 18,10 \times 150 \mathrm{~mm}$ ) eluted at a flow rate of $2.5 \mathrm{~mL} \mathrm{~min}^{-1}$ with aqueous acetonitrile as the mobile phase (0 - $20 \mathrm{~min}: 45 \%, 20$ 40 min: gradient to $100 \%, 40$ - $60 \mathrm{~min}$ : $100 \%$ acetonitrile).

Enzyme preparation and assay. Cultured cells ( $5 \mathrm{~g})$ of yeast WAT21 were homogenized with $0.1 \mathrm{M}$ Na phosphate buffer (pH 7.4) containing $1 \mathrm{mM}$ EDTA, $1 \mathrm{mM}$ DTT, $0.1 \mathrm{mM}$ PMSF, $15 \mathrm{mM}$ 2-mercaptoethanol, 15\% glycerol, $250 \mathrm{mM}$ sucrose, $40 \mathrm{mM}$ ascorbate, and 1\% insoluble polyvinylpolypyrrolidone. The homogenates were centrifuged for $10 \mathrm{~min}$ at $8,000 \times \mathrm{g}$, and the resultant supernatants were re-centrifuged for $30 \mathrm{~min}$ at $20,000 \times$ g. Cold acetone was then added to the obtained supernatants (final volume 40\%), and the acetone precipitates were re-suspended in $0.1 \mathrm{M} \mathrm{Na}$ phosphate buffer ( $\mathrm{pH} 7.4$ ) containing $1.5 \mathrm{mM} 2$-mercaptoethanol and $30 \%$ glycerol for crude enzyme solutions. After $30 \mathrm{~min}$ of incubation at $37^{\circ} \mathrm{C}$, the assay mixture was extracted with ethyl acetate $(1.2 \mathrm{~mL} \times 3)$. The obtained ethyl acetate soluble fractions were then concentrated and loaded onto a Sep-Pak C18 cartridge eluted with 0, 50, and $100 \%$ methanol (5 mL each). The $100 \%$ methanol fractions that evidenced biological activity in the rice lamina inclination assay were purified further via the same reversed-phase HPLC mentioned above.

GC-MS/SIM analysis. GC-MS/SIM analysis was performed a capillary GC-MS: a Hewlett-Packard 5973 mass spectrometer (Electron impact ionization, 70 electron voltage) coupled to 6890 gas chromatography fitted with a fused silica capillary column (HP-5, $0.25 \mathrm{~mm} \times 30 \mathrm{~m}, 0.25 \mu \mathrm{m}$ film thickness). The oven temperature was maintained at $175^{\circ} \mathrm{C}$ for $2 \mathrm{~min}$, elevated to $280{ }^{\circ} \mathrm{C}$ at a rate of $40{ }^{\circ} \mathrm{C} \mathrm{min}^{-1}$ and then maintained at $280{ }^{\circ} \mathrm{C}$. Helium was used as the carrier gas at a flow rate of $1 \mathrm{~mL} \mathrm{~min}^{-1}$ and samples were introduced using an on-column injection mode.

Acknowledgments. This research was supported by KOSEF/MEST (R01-2007-000-20074-0) and KRF (2006-C00149).

\section{References}

1. Gianfagna, T. J. In Plant Hormones: Physiology, Biochemistry and Molecular Biology; Davies, P. J., Eds.; Kluwer: Dordrecht, 1995; p 751.

2. Clouse, S. D.; Sasse, J. M. Annu. Rev. Plant Physiol. Plant Mol. Biol. 1998, 49, 427.

3. Wada, K.; Shingo Marumo, S.; Abe, H.; Morishita, T.; Nakmura, K.; Uchiyama, M.; Mori, K. Agric. Biol. Chem. 1984, 3, 719.

4. Bajguz, A.; Tretyn, A. Phytochemistry 2003, 62, 1027.

5. Kim, T.-W.; Chang, S. C.; Lee, J. S.; Takatsuto, S.; Yokota, T.; Kim, S.-K. Plant Physiol. 2004, 135, 1231.

6. Pompon, D.; Louerat, B.; Bronine, A.; Urban, P. Methods Enzymol. 1996, 272, 51 .

7. Duan, H.; Schuler, M. Phytochemistry 2006, 5, 507.

8. Kim, T.-W.; Hwang, J.-Y.; Kim, Y.-S.; Joo, S.-H.; Chang, S. C.; Lee, J.-S.; Takatsuto, S.; Kim, S.-K. Plant Cell 2005, 17, 2397.

9. Fujioka, S. In Brassinosteroids: Steroidal Plant Hormones; Sakurai, A., Yokota, T., Clouse, S. D., Eds.; Springer-Verlag: Tokyo, 1999; p 21.

10. Brian, P. W.; Elson, G. W.; Hemming, H. G.; Radley, M. J. Sci. Food. Agr 1954, 5, 602. 